\title{
Rough Interval Valued \\ Intuitionistic Fuzzy Ideals in $\Gamma$ \\ - Semigroups
}

Emperor Journal of Applied Scientific Research

ISSN No. 258I-964X(O)

202I, Vol. 3 Issue-05

(C) The Author(s) 2021

http://ejasr.mayas.info

\author{
V. S. Subha', P. Dhanalakshmi ${ }^{2}$ \\ 'Assistant Professor, Department of Mathematics, \\ Govt. Arts College, C.Mutlur, Chidambaram-608I02. \\ IResearch Scholar, Annamalai University.
}

\begin{abstract}
The spot of this paper is to present the opportunity of earth shattering stretch regarded intuitionistic cushioned fixations in $\Gamma$ a few its properties. We other than show the chance of repulsive freedom regarded intuitionistic cushioned left (right, two-sided, bi-,(I,2)- ) appraisals in $\mathrm{r}$ a couple of properties of these norms.. Index terms: Brutal reach regarded intuitionistic cushioned set, horrible spread regarded intuitionistic tricky left (right) inclines, savage stretch regarded intuitionistic warm bi-ideal, loathsome freedom considered intuitionistic satisfying $(I, 2)$ - ideal.
\end{abstract}

\section{Introduction}

Zadeh [I] introduced cushioned set as a focal mathematical instrument to depict and amassing the things whose cutoff is dull. Various experts have related themselves in enhancing the pieces of information and aftereffects of the unadulterated variable-based math to cushioned set. The upset set speculation began by Pawlak [7] is a beast mathematical model to oversee shadowiness and granularity to the level of approximations. Pawlak and Skowron [8] set up express levels of progress on savage sets to figure spaces. Kuroki introduced the opportunity of horrible guidelines in semigroups. Xiao development Zhang [14]studied unforgiving prime norms and disturbing woolen prime fights in semigroups. In 1986 Atanassov[I] presented the intuitionistic sensitive sets as theory of satisfying sets. Jayanta Ghosh et al. [ 4] introduced savage intuitionistic fragile principles in semigroups. Atanassov et al.[I,2] introduced range regarded intuitionistic warm sets, a hypothesis of both astonishing intuitionistic sets and stretch considered sensitive sets. A couple of experts applied field-saw intuitionistic cushioned sets to arithmetical new developments. Subha et al. [10,II] introduced the numerical plans of guaranteed appear at regarded cushioned sets. Subha et al. [12] surveyed the opportunity of shocking stretch kept cushioned standards in gamma-semigroups.

\section{Preliminaries}

This part deals with the immense definitions [I-16], which will be relied upon in this work. In this paper, nearby if disregarding passed on unequivocally, G continually loosens up a r-semigroup with character.

\section{Rough Interval Valued Intuitionistic Fuzzy Subsets (RIVIFS) In Г-Semigroup}

This section deals with RIVIF sets in $\Gamma$-semigroup. Throughout this paper let us denote $\pi$ as a congruence relation on $G_{x}$

Let $\pi$ be a congruence relation on $G$, ie., $\pi$ is an equivalence relation on $G$ such that $(a, b) \in \pi \Rightarrow(a y x, b y x) \in \pi$ and $(x y a, x y b) \in \pi \forall x \in G$.

For a congruence relation $\pi$ on $G$, we have $[a]_{\pi} \Gamma[b]_{\pi} \subseteq[a \Gamma b]_{\pi} \forall a, b \in G$. A congruence relation $\pi$ on $G$ is called complete if $[l]_{\pi} \Gamma[k]_{\pi}=[L k]_{\pi}$ for all $l, k \in G$.

Let $I$ be an IIF s of $G$. Then an IVIF, $\pi_{8}(J)=\left(\pi_{8}\left(\mu_{J}\right), \pi_{8}\left(v_{J}\right)\right)$ and $\pi^{8}(J)=\left(\pi^{8}\left(\mu_{J}\right), \pi^{8}\left(v_{J}\right)\right)$ are respectively called $\pi$-lower and $\pi$-upper approximation of $J$ where $\forall l \in G$,

$$
\begin{aligned}
& \pi_{s}\left(\mu_{J}\right)(l)=\bigwedge_{a \in[l]]_{\pi}} \mu_{J}(a), \quad \pi_{s}\left(v_{J}\right)(l)=V_{a \in[l]]_{\pi}} v_{J}(a) \text { and }
\end{aligned}
$$

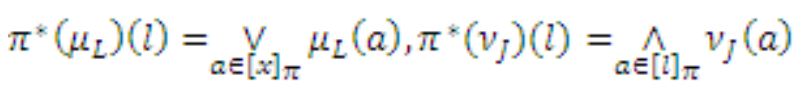

for an IVIF J of $G . \pi(J)=\left(\pi_{8}(J), \pi^{*}(J)\right)$ is called RIVIF set with respect to $\pi$ if $\pi_{8}(J) \neq \pi^{*}(J)$. 
Note: $\pi_{8}\left(\mu_{J}\right)(l)+\pi_{8}\left(v_{J}\right)(l)=\bigwedge_{a \in[l]]_{\pi}} \mu_{J}(a)+\underset{a \in[l]]_{\pi}}{v} v_{J}(a) \leq \bigwedge_{a \in[l]]_{\pi}} \mu_{J}(a)+\underset{a \in[l]]_{\pi}}{ }\left(1-\mu_{J}(a)\right)$

$$
=\bigwedge_{a \in[1] e} \mu_{J}(a)+1-\bigwedge_{a \in[1] e} \mu_{J}(a)=1
$$

Similarly $\pi^{*}\left(\mu_{J}\right)(x)+\pi^{*}\left(v_{J}\right)(x) \leq 1$ for all $l \in G$. This proves that $\pi_{s}(J)=\left(\pi_{*}\left(\mu_{J}\right), \pi_{s}\left(v_{J}\right)\right)$ and $\pi^{*}(J)=\left(\pi^{*}\left(\mu_{J}\right), \pi^{*}\left(v_{J}\right)\right)$ are IVIF set of $G$.

Theorem.3.I. Let $\pi$ and $\varphi$ be two congruence relation of $G$. If $/$ and $K$ are any two IVIF sets of $G$, then the following conditions are holds:
(i) $\pi_{8}(\mathrm{~J}) \subseteq \mathrm{J} \subseteq \pi^{8}(\mathrm{~J})$
(ii) $\pi_{8}\left(\pi_{8}(\mathrm{O})\right)=\pi_{8}(\mathrm{O})$
(iii) $\pi^{8}\left(\pi^{8}(\mathrm{~J})\right)=\pi^{8}(\mathrm{~J})$
(iv) $\pi^{*}\left(\pi_{8}(\mathrm{~J})\right)=\pi_{8}(\mathrm{~J})$
(v) $\pi_{8}\left(\pi^{8}(\mathrm{~J})\right)=\pi^{8}(\mathrm{~J})$
(vi) $\left(\pi^{8}\left(J^{c}\right)\right)^{c}=\pi_{8}(\mathrm{~J})$
(vii) $\left(\pi_{*}\left(J^{c}\right)\right)^{c}=\pi^{*}(\mathrm{~J})$
(viii) $\pi_{8}(\mathrm{~J} \cap \mathrm{K})=\pi_{8}(\mathrm{~J}) \cap \pi_{8}(\mathrm{~K})$
(ix) $\pi^{8}(\mathrm{~J} \cap \mathrm{K})=\pi^{8}(\mathrm{~J}) \cap \pi^{8}(\mathrm{~K})$
(x) $\pi^{8}(\mathrm{~J} \cup \mathrm{K})=\pi^{8}(\mathrm{~J}) \cup \pi^{8}(\mathrm{~K})$
(xi) $\pi_{8}(\mathrm{JU} \mathrm{K}) \supseteq \pi_{8}(\mathrm{~J}) \cup \pi_{8}(\mathrm{~K})$
(xii) $I \subseteq K \Rightarrow \pi_{8}(\mathrm{O}) \subseteq \pi_{8}(\mathrm{~K})$
(xii) $I \subseteq K \Rightarrow \pi^{*}(\mathrm{~J}) \subseteq \pi^{*}(\mathrm{~K})$
(xiv) $\theta \subseteq \varphi \Rightarrow \pi_{8}(\mathrm{~J}) \supseteq \varphi_{8}(\mathrm{~J})$
(xv) $\theta \subseteq \varphi \Rightarrow \pi^{*}(\mathrm{~J}) \subseteq \varphi^{*}(\mathrm{~J})$

Theorem.3.2. Let $\pi$ be a congruence relation of $G$. If $/$ and $K$ are any two IVIF of $G$, then $\pi^{8}(J) \circ \pi^{*}(K) \subseteq \pi^{8}(J \circ K)$.

Proof: Let $I=\left(\mu_{j}, v_{J}\right), K=\left(\mu_{K}, v_{K}\right)$ be any two IVIF of $G$. Then

$$
\begin{aligned}
& \pi^{*}(J) \circ \pi^{*}(K)=\left(\pi^{*}\left(\mu_{J}\right) \bullet \pi^{*}\left(\mu_{K}\right), \pi^{*}\left(v_{J}\right) \circ \pi^{*}\left(v_{K}\right)\right) \text { and } \\
& \pi^{*}(J \circ K)=\left(\pi^{*}\left(\mu_{J} \bullet \mu_{K}\right), \pi^{*}\left(v_{J} \bullet v_{K}\right) .\right.
\end{aligned}
$$

To show $\pi^{8}(J) \circ \pi^{8}(K) \subseteq \pi^{8}(J \circ K)$, for that we have to prove that for all $l \in G$

$$
\begin{aligned}
& \left(\pi^{8}\left(\mu_{J}\right) \bullet \pi^{*}\left(\mu_{K}\right)\right)(l) \leq \pi^{*}\left(\mu_{J} \bullet \mu_{K}\right)(l) \text { and } \\
& \left(\pi^{*}\left(v_{J}\right) \bullet \pi^{*}\left(v_{K}\right)(l) \geq \pi^{*}\left(v_{J} \bullet v_{K}\right)(l) .\right.
\end{aligned}
$$

Now for all $l \in G$

$$
\begin{aligned}
\left(\pi^{*}\left(\mu_{J}\right) \odot \pi^{*}\left(\mu_{K}\right)\right)(l)= & V_{I=p y q}\left[\pi^{*}\left(\mu_{J}\right)(p) \wedge \pi^{*}\left(\mu_{K}\right)(q)\right] \\
& =V_{I=p y q}\left[\left(V_{a \in[p]_{\pi}} \mu_{J}(a)\right) \wedge\left(V_{b \in[q]_{\pi}} \mu_{K}(b)\right)\right] \\
& =V_{I=p y q}\left[V_{a \in[p]_{\pi} b \in[q]_{\pi}}\left(\mu_{J}(a) \wedge \mu_{K}(b)\right)\right]
\end{aligned}
$$




$$
\begin{aligned}
& \leq V_{I=p y q}\left[V_{a y b \in[p y q]_{\theta}}\left(\mu_{J}(a) \wedge \mu_{K}(b)\right)\right], \\
& =V_{a y b \in[1] \pi}\left(\mu_{J}(a) \wedge \mu_{K}(b)\right) \text {, } \\
& =V_{\alpha \in[t]]_{\pi} \alpha=a y b}\left(\mu_{J}(a) \wedge \mu_{K}(b)\right) \\
& =V_{\propto \in[l]]_{\pi}} V_{\propto=a \gamma b}\left(\mu_{J}(a) \wedge \mu_{K}(b)\right) \\
& =V_{\alpha \in[\mathbb{[}]_{\pi}}\left(\mu_{J} \circ \mu_{K}\right)(\alpha) \\
& =\pi^{*}\left(\mu_{J} \odot \mu_{K}\right)(l)
\end{aligned}
$$

Again,

$$
\begin{aligned}
& \left(\pi^{*}\left(v_{J}\right) \otimes \pi^{*}\left(v_{K}\right)\right)(l)=\Lambda_{I=p y q}\left[\pi^{*}\left(v_{J}\right)(p) \vee \pi^{*}\left(v_{K}\right)(q)\right] \\
& =\Lambda_{I=p y q}\left[\left(\Lambda_{a \in[p]_{\pi}} v_{J}(a)\right) \vee\left(\Lambda_{b \in[q]_{\pi}} v_{K}(b)\right)\right] \\
& =\Lambda_{\mathrm{I}}=\mathrm{pyq}_{\mathrm{q}}\left[\Lambda_{\mathrm{aE}}[\mathrm{g}]_{\pi}, b \in[z]_{\pi}\left[v_{J}(a) \vee v_{K}(b)\right]\right] \\
& \geq \Lambda_{\mathrm{I}=p y q}\left[\Lambda_{a y b \in[p y q]_{\pi}}\left(v_{J}(a) \vee v_{K}(b)\right)\right] \\
& =\Lambda_{a y b \in[l]}\left(v_{J}(a) \vee v_{K}(b)\right) \text {, } \\
& =\bigwedge_{\alpha \in[a]]_{\pi} \alpha=a \gamma b}\left(v_{J}(a) \vee v_{K}(b)\right) \\
& =\Lambda_{\alpha \in[x]_{\pi}} \Lambda_{\propto=a y b}\left(v_{J}(a) \vee v_{K}(b)\right) \\
& =\Lambda_{\alpha \in[\alpha]_{\pi}}\left(v_{J} \circ v_{K}\right)(\alpha)=\pi^{8}\left(v_{J} \bullet v_{K}\right)
\end{aligned}
$$

Thus we have $\pi^{8}(J) \circ \pi^{8}(K) \subseteq \pi^{8}(J \circ K)$.

Theorem.3.3. Let $\pi$ be a congruence relation of $G$. If /and $K$ are any two IVIF set of $G$, then $\pi_{*}(J) \circ \pi_{*}(K) \subseteq \pi_{*}(J \circ K)$.

Proof: Since $\pi$ is complete congruence relation on $G$. Let $I=\left(\mu_{J}, v_{J}\right), K=\left(\mu_{K}, v_{K}\right)$ be any two $I I F$ of $G$.

Then

$$
\begin{aligned}
& \pi_{8}(J) \circ \pi_{8}(K)=\left(\pi_{8}\left(\mu_{J}\right) \odot \pi_{8}\left(\mu_{K}\right), \pi_{8}\left(v_{J}\right) \odot \pi_{8}\left(v_{K}\right)\right) \text { and } \\
& \pi_{i}(J \circ K)=\left(\pi_{i}\left(\mu_{J} \circ \mu_{K}\right), \pi_{s}\left(v_{J} \circ v_{K}\right)\right. \text {. }
\end{aligned}
$$

To show $\pi_{*}(J) \circ \pi_{*}(K) \subseteq \pi_{*}(J \circ K)$. We have to prove that for all $l \in G$

$$
\begin{aligned}
& \left(\pi_{s}\left(\mu_{J}\right) \cdot \pi_{s}\left(\mu_{K}\right)\right)(l)=\Lambda_{\mathrm{L}=p y q}\left[\pi_{s}\left(\mu_{J}\right)(p) \vee \pi_{s}\left(\mu_{K}\right)(q)\right] \\
& =\Lambda_{\mathrm{I}=p \gamma q}\left[\left(\Lambda_{a \in[p]_{\pi}} \mu_{J}(a)\right) \vee\left(\Lambda_{b \in[q]_{\pi}} \mu_{K}(b)\right)\right] \\
& =\Lambda_{I=p y q}\left[\Lambda_{\left.a \in[y]_{\pi} b[z]_{\mu}\right]_{I}}\left[\mu_{J}(a) \vee \mu_{K}(b)\right]\right] \\
& \geq \Lambda_{I=p y q}\left[\Lambda_{a y b \in[p y q]_{\pi}}\left(\mu_{J}(a) \vee \mu_{K}(b)\right)\right] \\
& =\bigwedge_{a y b \in[]_{\pi}}\left(\mu_{J}(a) \vee \mu_{K}(b)\right), \\
& =\bigwedge_{\alpha \in[x]_{\pi \propto}=a y b}\left(\mu_{J}(a) \vee \mu_{K}(b)\right) \\
& =\Lambda_{\alpha \in[a]]_{\mathbb{M}}} \Lambda_{\propto=a y b}\left(\mu_{J}(a) \vee \mu_{K}(b)\right) \\
& =\Lambda_{\alpha \in[x]_{\pi}}\left(\mu_{J} \circ \mu_{K}\right)(\alpha)=\pi^{*}\left(\mu_{J} \circ \mu_{K}\right)
\end{aligned}
$$


Again,

$$
\begin{aligned}
& \left(\pi_{s}\left(v_{J}\right) \circ \pi_{s}\left(v_{K}\right)\right)(l)=\Lambda_{I=p y q}\left[\pi_{s}\left(v_{J}\right)(p) \vee \pi_{s}\left(v_{K}\right)(q)\right] \\
& =\Lambda_{I=p y q}\left[\left(\Lambda_{a \in[p]_{\pi}} v_{J}(a)\right) \vee\left(\Lambda_{b \in[q]_{\pi}} v_{K}(b)\right)\right] \\
& =\Lambda_{\mathrm{I}=p y q}\left[\Lambda_{a \in[p]_{\pi} b \in[q]_{\pi}[}\left[v_{J}(a) \vee v_{K}(b)\right]\right] \\
& \geq \Lambda_{I=p y q}\left[\Lambda_{a y b \in[p y q]_{\pi}}\left(v_{J}(a) \vee v_{K}(b)\right)\right] \text {, } \\
& \left.=\Lambda_{a y b \in[}\right]_{\pi}\left(v_{J}(a) \vee v_{K}(b)\right) \text {, } \\
& =\bigwedge_{\alpha \in[l]]_{\pi} \alpha=a y b}\left(v_{J}(a) \vee v_{K}(b)\right) \\
& =\wedge_{\alpha \in[l]]_{\pi}} \wedge_{\alpha=a y b}\left(v_{J}(a) \vee v_{K}(b)\right) \\
& =\bigwedge_{\propto \in[1]}\left(v_{I} \circ v_{K}\right)(\alpha)=\pi_{s}\left(v_{L} \circ v_{K}\right)
\end{aligned}
$$

Thus we have $\pi_{*}(J) \circ \pi_{*}(K) \subseteq \pi_{*}(J \circ K)$.

Theorem .3.4. Let $\pi$ and $\varphi$ be two congruence relation of $G$. If $/$ is an IVIF set of $G$ then $(\pi \cap \varphi)^{*}(J) \subseteq \pi^{*}(J) \cap \varphi^{*}(J)$.

Proof: We know that $\pi \cap \varphi$ is also a congruence relation on $G$. Also $\pi \cap \varphi \subseteq \pi$ and $\pi \cap \varphi \subseteq \varphi$. Let $/$ be an IVIF set of $G$. Then by using Theorem 3.2(15) we obtain $(\pi \cap \varphi)^{8}(L) \subseteq \pi^{*}(L)$ and $(\pi \cap \varphi)^{*}(L) \subseteq \varphi^{*}(L)$. Then we have $(\pi \cap \varphi)^{*}(L) \subseteq \pi^{*}(L) \cap \varphi^{*}(L)$.

Theorem .3.5 Let $\pi$ and $\varphi$ be two congruence relation of $G$. If $L$ is an IVIF of $G$ then $(\pi \cap \varphi)_{8}(L) \subseteq \pi_{8}(L) \cap \varphi_{8}(L)$.

Proof: It is easily to observe that $\pi \cap \varphi$ is also a congruence relation on $G$. Also $\pi \cap \varphi \subseteq \pi$ and $\pi \cap \varphi \subseteq \varphi$.

Let $L$ be an IVIF set of $G$. Then by using Theorem 3.2(14) we obtain $(\pi \cap \varphi)_{8}(L) \subseteq \pi_{8}(L)$ and $(\pi \cap \varphi)_{s}(L) \subseteq \varphi_{8}(L)$. Therefore $(\pi \cap \varphi)_{s}(L) \subseteq \pi_{8}(L) \cup \varphi_{8}(L)$.

\section{Rough Interval Valued Intuitionistic Fuzzy (Rivifi) Ideals Of $\Gamma$ -} Semigroup

This section introduces the notion of ideal, bi-ideal, and $(1,2)$-ideal. Then, more exciting properties are proved.

Definition.4.I Let $\pi$ be a congruence relation on $M$. An IVIF / of $G$ is called an upper (lower)-RIVIF sub $\Gamma$-semigroup of $G$ if $\pi_{8}(J)\left(\pi_{8}(J)\right)$ is an IVIF sub- $\Gamma$-semigroup of $G$.

$J$ is called a RIVIF sub- $\Gamma$-semigroup of $G$ if it is both upper and lower-RIVIF sub- $Г$ semigroup of $G$. 
Theorem.4.2. Let $\pi$ be a congruence relation on $G$. If $I$ is an IVIF sub-Г-semigroup of $G$, then $L$ is an upper RIVIF sub- $\Gamma$-semigroup of $G$.

Proof: Since $/$ is an $I V I F$ sub- $\Gamma$-semigroup of $G$ and $l, k \in G$ and $\gamma \in \Gamma$.

Then

$$
\begin{aligned}
& \pi^{*}\left(\mu_{J}\right)(l y k)=V_{p \in[l y k]_{\pi}} \mu_{J}(p) \\
& \geq V_{p \in[l]]_{\pi v}[k]_{\pi}} \mu_{J}(p) \\
& =V_{q y r}\left[[]_{\pi V}[k]_{\pi} \mu_{I}(q Y r)\right. \\
& \geq \mathrm{V}_{\mathrm{q} \in[\mathrm{l}]_{\pi} r \in[k]_{\pi}}\left[\mu_{J}(q) \wedge \mu_{I}(r)\right] \\
& =V_{q \in[k]_{\pi}} \mu_{J}(q) \wedge V_{r \in[k]_{\pi}} \mu_{J}(r) \\
& =\pi^{*}\left(\mu_{J}\right)(l) \wedge \pi^{*}\left(\mu_{J}\right)(k) . \\
& \pi^{8}\left(v_{J}\right)(l y k)=\Lambda_{p \in[l y k]_{\pi}} v_{J}(p) \\
& \leq \Lambda_{p \in[t]_{\pi \gamma}[k]_{\pi} v_{J}(z)} \\
& =\bigwedge_{q Y r \in[1]]_{\pi} \gamma[k]_{\pi} v_{J}(q \gamma r)}
\end{aligned}
$$

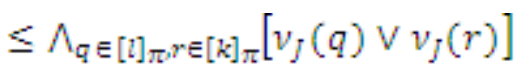

$$
\begin{aligned}
& =\bigwedge_{q \in[l]]_{\pi}} v_{J}(q) \vee \Lambda_{r \in[k]_{\pi}} v_{J}(r) \\
& =\pi^{*}\left(v_{J}\right)(l) \vee \pi^{*}\left(v_{J}\right)(k)
\end{aligned}
$$

Hence $I$ is an upper RIVIF sub- $\Gamma$-semigroup of $G$.

Theorem.4.3. Let $\pi$ be a congruence relation on $G$. If $I$ is an IVIF sub $\Gamma$-semigroup of $G$, and if $\pi$ is complete then $J$ is a lower RIVIF sub $\Gamma$-semigroup of $G$.

Proof: Let / be an IVIF sub- $\Gamma$-semigroup of $G$ and $x_{y} y \in G$.

Then

$$
\begin{aligned}
& \pi_{8}\left(\mu_{J}\right)(l y k)=\Lambda_{p \in[l y k]_{\theta}} \mu_{J}(p) \\
& =\bigwedge_{p \in[t] \pi} v[k]_{\pi} \mu_{J}(p) \\
& =\bigwedge_{q Y r \in[}[]_{\pi \gamma}[k]_{\pi} \mu_{J}(q Y r)
\end{aligned}
$$

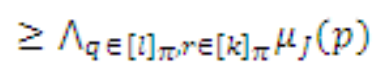

$$
\begin{aligned}
& =\Lambda_{\mathrm{q} \in[t]_{\pi}} \mu_{J}(q) \vee \Lambda_{r \in[k]_{\pi}} \mu_{J}(r) \\
& =\pi_{8}\left(\mu_{J}\right)(\mathrm{l}) \wedge \pi_{8}\left(\mu_{J}\right)(k) \\
& \pi_{s}\left(v_{P}\right)(l y k)=V_{p \in[l y k]_{\theta}} v_{J}(p) \\
& =V_{p \in[1]_{\pi V}[k]_{\pi} v_{J}}(p) \\
& =V_{q Y r \in[R]_{\pi} V[k]_{\pi}} v_{J}(q Y r)
\end{aligned}
$$

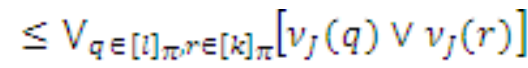

$$
\begin{aligned}
& =\mathrm{V}_{\mathrm{q} \in[\mathrm{l}]_{\pi}} v_{J}(q) \vee \mathrm{V}_{r \in[k]_{\pi}} v_{J}(r) \\
& =\pi_{s}\left(v_{J}\right)(l) \vee \pi_{s}\left(v_{J}\right)(k) \text {. }
\end{aligned}
$$

This shows that $\pi_{s}(\mathrm{I})$ is an IVIF sub- $\Gamma$-semigroup of $G$. 
Definition.4.4 Let $\pi$ be a congruence relation on M. An IVIF / of $G$ is called an upper (lower)RIVIF left(right, two-sided)-ideal of $G$ if $\pi_{s}(J)\left(\pi_{s}(J)\right)$ is an IVIF left(right, two-sided)ideal of $G$.

$J$ is called a RIVIF left(right, two-sided)-ideal of $G$ if it is both upper and lower-RIVIF left(right, two-sided)-ideal of $G$.

Theorem.4.5. Let $\pi$ be a congruence relation on $M$. If $I$ is an IVIF left(right,twosided)ideal of $G$, then

(i) $\quad J$ is an upper RIVIF left (right,two-sided) ideal of $G$.

(ii) If $\pi$ is complete,then $/$ is lower RIVIF left(right,two-sided)ideal of $G$.

Proof: Let $I$ be an IVIF left ideal of $G_{x} g_{x} h \in G$ and $\gamma \in \Gamma$.

(i) $\pi^{*}\left(\mu_{J}\right)(l y k)=V_{p \in[l y k]_{\pi}} \mu_{J}(p)$

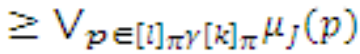

$$
\begin{aligned}
& =V_{q Y r E[}[]_{\pi Y}[k]_{\pi} \mu_{J}(q Y r) \\
& \geq \mathrm{V}_{r \in[k]_{\pi} \mu_{J}} \mu_{J}(r) \\
& =\pi^{*}\left(\mu_{J}\right)(k) \text {. } \\
& \pi^{*}\left(v_{J}\right)(l y k)=\Lambda_{\mathrm{p} \in[l y k]_{\pi}} v_{J}(p) \\
& \leq \Lambda_{p \in[z]_{\pi} \gamma[k]_{\pi}} v_{J}(z) \\
& \left.=\bigwedge_{q Y r E[t}\right]_{\pi V}[k]_{\pi} v_{J}(q \gamma r) \\
& \leq \bigwedge_{r \in[k]_{\pi}} v_{J}(r) \\
& =\pi^{*}\left(v_{J}\right)(k)
\end{aligned}
$$

This shows that $\pi^{*}(I)$ is an IVIF left ideal of $G$. Therefore $/$ is an upper RIVIF left ideal of $G$. Similarly we can prove the other cases.

(ii)Similar to (i).

Definition.4.6 Let $\pi$ be a congruence relation on $G$. An IVIF set $B$ of $G$ is called an upper (lower)-RIVIF bi-ideal of $G$ if $\pi_{s}(B)\left(\pi_{s}(B)\right)$ is an IVIF bi-ideal of $G . B$ is called a RIVIF bi-ideal of $G$ if it is both upper and lower-RIVIF bi-ideal of $G$.

Theorem.4.8. Let $\pi$ be a congruence relation on $G$. If $I$ is an IVIF bi-ideal of $G$, then $I$ is an upper RIVIF bi-ideal of $G$.

Proof: Let $I$ be an IVIF bi-ideal of $G$ and $l_{p} m, n \in G$ and $\alpha, \beta \in \Gamma$. Then

$$
\begin{aligned}
& \left.\pi^{*}\left(\mu_{I}\right)(\operatorname{lam} \beta n)=V_{\mathrm{pE}[\operatorname{lam} \beta, \beta n}\right]_{\pi} \mu_{I}(p)
\end{aligned}
$$

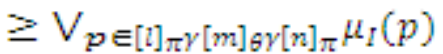

$$
\begin{aligned}
& =V_{q \alpha r \beta s \in[l]]_{\pi} \gamma[m]_{\pi} \gamma[n]_{\pi}} \mu_{I}(q \alpha r \beta s) \\
& \geq V_{q \in[q]_{\pi} r \in[m]_{\pi^{s}} s[n]_{\Pi}}\left[\mu_{I}(q) A \mu_{I}(s)\right]
\end{aligned}
$$




$$
\begin{aligned}
& =V_{q \in[t]_{\pi} s \in[n]_{\pi}}\left[\mu_{I}(q) \wedge \mu_{I}(s)\right] \\
& =V_{q \in[l]_{\pi}} \mu_{I}(q) \wedge V_{s \in[n]_{\pi}} \mu_{I}(s) \\
& =\pi^{*}\left(\mu_{P}\right)(l) \wedge \pi^{*}\left(\mu_{I}\right)(n) .
\end{aligned}
$$

Similarly we can prove $\pi^{*}\left(v_{I}\right)(\operatorname{lom} \beta n) \leq \pi^{*}\left(v_{I}\right)(l) \vee \pi^{*}\left(v_{I}\right)(n)$. Hence $\pi^{*}(I)$ is an IVIF bi-ideal of $G$. Therefore I is an upper RIVIF bi-ideal of $G$.

By similar arguments we can prove the following theorem.

Theorem.4.9. Let $\pi$ be a congruence relation on $M$. If $I$ is an IVIF bi-ideal of $G$, then $I$ is an lower RIVIF bi-ideal of $G$.

Definition.4.4 Let $\pi$ be a congruence relation on M. An IVIF set $/$ of $G$ is called an upper (lower)-RIVIF $(1,2)$-ideal of $G$ if $\pi_{8}(J)\left(\pi_{8}(J)\right)$ is an IVIF $(1,2)$-ideal of $G . I$ is called a RIVIF $(1,2)$-ideal of $G$ if it is both upper and lower-RIVIF $(1,2)$-ideal of $G$.

Theorem.4.10. Let $\pi$ be a congruence relation on $M$. If $I$ is an $i$ IVIF $(1,2)$-ideal of $G$, then it is an upper RIVIF $(1,2)$-ideal of $G$.

Proof: Proof is similar to Theorem 4.8.

Theorem.4.II. Let $\pi$ be a congruence relation on M. If $I$ is an $i$ IVIF $(1,2)$-ideal of $G$, then it is an lower RIVIF $(1,2)$-ideal of $G$.

Proof: Proof is similar to Theorem 4.9.

Theorem.4.12. Let $\pi$ be a congruence relation on $M$. If $L$ and $M$ are IVIF left ideal and IVIF right ideal of $G$ respectively. Then $\pi^{*}(L \circ M) \subseteq \pi^{*}(L) \cap \pi^{*}(M)$.

Proof: Let $L$ and $M$ be IVIF left ideal and IVIF right ideal of $G$, respectively.

Then $\pi^{*}(L \circ M)=\left(\pi^{*}\left(\mu_{L} \circ \mu_{M}\right), \pi^{*}\left(v_{L} \circ v_{M}\right)\right.$ and

$$
\pi^{*}(L) \cap \pi^{*}(M)=\left(\pi^{*}\left(\mu_{L}\right) \cap \pi^{*}\left(\mu_{M}\right), \pi^{*}\left(v_{L}\right) \cup \pi^{*}\left(v_{M}\right)\right) .
$$

To prove $\pi^{*}(L \circ M) \subseteq \pi^{*}(L) \cap \pi^{*}(M)$.

For that we have to prove for all $k \in G$ and $\gamma \in \Gamma$,

$$
\begin{aligned}
& \pi^{*}\left(\mu_{L} \bullet \mu_{M}\right)(k) \leq\left(\pi^{*}\left(\mu_{L}\right) \cap \pi^{*}\left(\mu_{M}\right)\right)(k) \text { and } \\
& \pi^{*}\left(v_{L} \bullet v_{M}\right)(k) \geq\left(\pi^{*}\left(v_{L}\right) \cap \pi^{*}\left(v_{M}\right)\right)(k) .
\end{aligned}
$$

Now for all $k \in G$,

$$
\begin{aligned}
& \pi^{*}\left(\mu_{L} \circ \mu_{M}\right)(k)=V_{n \in[k]_{M}}\left(\mu_{L} \circ \mu_{M}\right)(n) \\
& =V_{n \in[k]_{\pi}} V_{n=p y q}\left[\mu_{L}(p) \wedge \mu_{M}(q)\right] \\
& =V_{n \in[k]]_{M}}\left[\mu_{L}(p) \wedge \mu_{M}(q)\right] \\
& \leq V_{n \in[k]_{\pi} \in[k]_{M}}\left[\mu_{L}(p) \wedge \mu_{M}(q)\right] \\
& =V_{n \in[k]_{\pi}} \mu_{L}(p) \wedge V_{m E[k]_{\pi}} \mu_{M}(q) \\
& =\pi^{*}\left(\mu_{L}\right)(k) \wedge \pi^{*}\left(\mu_{M}\right)(k) \\
& =\left(\pi^{*}\left(\mu_{L}\right) \cap \pi^{*}\left(\mu_{M}\right)\right)(k)
\end{aligned}
$$

Similarly we have to prove $\pi^{8}\left(v_{L} \otimes v_{M}\right)(k) \geq\left(\pi^{8}\left(v_{L}\right) \cap \pi^{*}\left(v_{M}\right)\right)(k)$. 
Hence $\pi^{*}(L \circ M) \subseteq \pi^{*}(L) \cap \pi^{*}(M)$.

Theorem.4.13. Let $\pi$ be a congruence relation on $M$. If $L$ and $M$ are IVIF left ideal and IVIF right ideal of $G$ respectively. Then $\pi_{8}(L \circ M) \subseteq \pi_{8}(L) \cap \pi_{8}(M)$.

Proof: Let $L$ and $M$ be IVIF left ideal and IVIF right ideal of $G$, respectively.

Then $\quad \pi_{s}(L \circ M)=\left(\pi_{s}\left(\mu_{L} \circ \mu_{M}\right), \pi_{s}\left(v_{L} \circ v_{M}\right)\right.$ and

$$
\pi_{8}(L) \cap \pi_{8}(M)=\left(\pi_{8}\left(\mu_{L}\right) \cap \pi_{s}\left(\mu_{M}\right), \pi_{8}\left(v_{L}\right) \cup \pi_{8}\left(v_{M}\right)\right) \text {. }
$$

To show that $\pi_{8}(L \circ M) \subseteq \pi_{8}(L) \cap \pi_{8}(M)$ we have to prove for all $k \in G$ and $\gamma \in \Gamma$,

$$
\begin{aligned}
& \pi_{s}\left(\mu_{L} \circ \mu_{M}\right)(k) \leq\left(\pi_{s}\left(\mu_{L}\right) \cap \pi_{8}\left(\mu_{M}\right)\right)(k) \text { and } \\
& \pi_{s}\left(v_{L} \circ v_{M}\right)(k) \geq\left(\pi_{8}\left(v_{L}\right) \cap \pi_{s}\left(v_{M}\right)\right)(k) .
\end{aligned}
$$

Now for all $k \in G$,

$$
\begin{aligned}
& \pi_{s}\left(\mu_{L} \odot \mu_{M}\right)(x)=\bigwedge_{n \in[k]_{M}}\left(\mu_{L} \odot \mu_{M}\right)(n) \\
& =\Lambda_{\mathrm{r} \in[k]_{\pi}} V_{n=p y q}\left[\mu_{L}(p) \wedge \mu_{M}(q)\right] \\
& =\bigwedge_{\mathrm{n} \in[k]_{M}}\left[\mu_{L}(p) \wedge \mu_{M}(q)\right] \\
& =\bigwedge_{\left.\mathrm{n} E[k]_{\pi}\right]_{L}} \mu_{\mathrm{L}}(p) \wedge \bigwedge_{\mathrm{n} \in[k]_{\pi} \mu_{M}}(q) \\
& =\pi_{s}\left(\mu_{L}\right)(k) \wedge \pi_{s}\left(\mu_{M}\right)(k) \\
& =\left(\pi_{s}\left(\mu_{L}\right) \cap \pi_{s}\left(\mu_{M}\right)\right)(k)
\end{aligned}
$$

Similarly we can prove $\pi_{s}\left(v_{L} \circ v_{M}\right)(x) \geq\left(\pi_{s}\left(v_{L}\right) \cap \pi_{s}\left(v_{M}\right)\right)(x)$.

Hence $\pi_{*}(L \circ M) \subseteq \pi_{*}(L) \cap \pi^{*}(M)$.

\section{CONCLUSION}

In this paper, we introduced the chance of vindictive reach regarded intuitionistic cushioned sets. Likewise, study the logarithmic properties of disturbing substances considered intuitionistic cushioned areas. Other than some frustrating properties of an unforgiving substance, regarded intuitionistic cushioned standards in r-semigroup are surveyed. Later on, we discharge up this longing to propose semigroup, suggested r-semigroup, etc

\section{REFERENCES}

I. K,T.Atanassav, Intuitionistic Fuzzy Sets, Fuzzy Sets And Systems, 20 (1986), 87-96.

2. K.T. Atanassov, G. Gargov, Interval valued intuitionistic fuzzy sets, Fuzzy Sets and Systems 3I (I 989) 343-349.

3. D.Dubois and H.Parade, Rough Fuzzy Sets And Fuzzy Rough Sets, International Journal of General Systems, 17 (1990), 191-209.

4. Jayantha Ghosh and T.K.Samatha, Rough Intuitionistic Fuzzy Ideals In Semigroups, Annals of Fuzzy Mathematics, Vol 4, No I, July 20I2, I55-168.

5. K.H.Kim and Y.B.Jun, Intuitionistic Fuzzy Ideals of Semigroups, Indian Journal of Pure and applied Mathematics, 33 (4) (2002), 443-449.

6. N. Kuroki, Rough Ideals In Semigroup, Information Sciences, 100 (1997), I30-163.

7. Z. Pawlak, Rough Sets And Fuzzy Sets, Fuzzy Sets And Systems, 17 (I) (1985), 99- 102.

8. Z. Pawlak and A. Showron, Rough Sets:Some Extensions, Information Sciences, 177(I) (2007) 28-40.

9. Z. Pawlak, Rough Sets-Theoretical Aspects Of Reasoning About Data, Kuluwer Academic publishers, Dordrecht(|99|).

10. V.S.Subha, N. Thillaigovindan and P.Dhanalakshmi , Interval valued rough fuzzy ideals in semigroups, Journal of Emerging Technlogies and Innovative Research,6(3),(20I9)27I-276.

II. V.S.Subha, N. Thillaigovindan and P.Dhanalakshmi, Interval valued rough fuzzy prime bi-ideals in semigroups, AIP Conference Proceedings(2019).

12. V.S.Subha and P.Dhanalakshmi, Rough Approximations of Interval rough fuzzy ideals in Gamma-semigroups, Annals of Communications in Mathematics, 3(4)(2020), 326-332. 
13. V.S. Subha, Rough intuitionistic fuzzy ideals in $\Gamma$ - semigroups, International Journal of Applied and advanced Scientific Research ,Vol I(I)(2016) 254-260.

14. Q M Xiao and Z L Zhang, Rough prime ideals and rough fuzzy prime ideals in semigroups, Information Sciences, 176(6), (2006), 725-733.

15. L.A.Zadeh, Fuzzy Sets, Information Control, 8,(1965) 338-353.

16. L.A.Zadeh, The Role Of Fuzzy Logic In The Management Of Uncertainity In Expert Systems, Fuzzy Sets And Systems, II(I),(1983) 197-198. 